\title{
Mapeamento do excesso de peso em gestantes e prematuridade: uma revisão de
}

\section{escopo}

\author{
Mapping overweight in pregnant women and prematurity: a scope review \\ Mapeo del exceso de peso en mujeres embarazadas y la prematuridad: una revisión del alcance
}

Recebido: 23/09/2021 | Revisado: 01/10/2021 | Aceito: 05/10/2021 | Publicado: 09/10/2021

Fabiana de Araújo Santos Rocha ${ }^{1}$ ORCID: https://orcid.org/0000-0003-0210-7453 Universidade Federal de São João del-Rei, Brasil E-mail: fabianarocha04@yahoo.com.br

Maryana Vieira Rodrigues ${ }^{2}$ ORCID: https://orcid.org/0000-0003-1816-9501 Universidade Federal de São João del-Rei, Brasil E-mail: maryanaufsj@gmail.com

Isabela Cristina Tomé ${ }^{3}$

ORCID: https://orcid.org/0000-0003-1642-5081 Universidade Federal de São João del-Rei, Brasil E-mail: isabelacristinatome@gmail.com

Thays Lopes de Almeida ${ }^{4}$ ORCID: https://orcid.org/0000-0002-5073-2862 Universidade Federal de São João del-Rei, Brasil

E-mail: thayslopesdealmeida5@gmail.com

Dayse Bazílio Rosa de Souza ${ }^{5}$ ORCID: https://orcid.org/0000-0003-4879-5378 Universidade Federal de São João del-Rei, Brasil E-mail: baziliodayse@gmail.com

Virgínia Junqueira Oliveira ${ }^{6}$ ORCID: https://orcid.org/0000-0003-1151-7673 Universidade Federal de São João del-Rei, Brasil E-mail: virginiaenf@ufsj.edu.br Márcia Christina Caetano Romano ${ }^{7}$ ORCID: https://orcid.org/0000-0002-1819-4689 Universidade Federal de São João del-Rei, Brasil E-mail: marciachristinacs@ufsj.edu.br

\begin{abstract}
Resumo
Introdução: O excesso de peso gestacional pode trazer implicações para a saúde da gestante e do concepto, e a prematuridade é um desfecho importante, pois apesar das tecnologias em assistência neonatal estarem cada vez mais avançadas, o recém-nascido prematuro pode necessitar de assistência hospitalar por complicações, além de ter maior risco de morte. Objetivo: Identificar e sintetizar evidências científicas sobre a associação entre obesidade durante a gestação e a prematuridade. Método: Trata-se de scoping review, desenvolvida com base nas recomendações do guia internacional PRISMA-ScR e no método proposto pelo Joanna Briggs Institute, Reviewers Manual 2017. Realizou-se pesquisa nas bases de dados eletrônicas estabelecidas. A coleta de dados ocorreu de outubro/2020 a abril/2021. Resultados: Recuperou-se um total de 5140 registros, sendo selecionados 42 estudos. Destes, 27 (65,85\%) apresentaram associação estatisticamente significativa entre excesso de peso e prematuridade. $O$ baixo peso da gestante também foi associado à prematuridade em $27 \%$ das investigações. Conclusão: Alterações nutricionais na gravidez, em especial, a obesidade, e também o baixo peso são elementos importantes na determinação do parto prematuro e devem ser considerados no acompanhamento pré-natal.
\end{abstract}

Palavras-chave: Obesidade; Excesso de peso; Prematuridade; Gestantes.

\begin{abstract}
Introduction: excessive gestacional weght can have implications for health of the pregnant woman and the fetus, and prematurity is important outcome, as despite technologies in neonatal care being increasingly advanced, premature newborns may need hospital care complications, in addition to having a higher risk of death. Objective: To identify and synthesize scientific evidence on the association between obesity during pregnancy and prematurity. Method: This is a scoping review, developed based on the recommendations of the international PRISMA-ScR guide and on the method proposed by the Joanna Briggs Institute, Reviewers Manual 2017. A search was carried out in the established electronic databases. Data collection took place from October/2020 to April/2021. Results: A total of 5140 records were retrieved, 42 studies were selected. Of these, $27(65.85 \%)$ showed a statistically significant association
\end{abstract}


between overweight and prematurity. Low birth weight in pregnant women was also associated with prematurity in $27 \%$ of the investigations. Conclusion: Nutritional changes in pregnancy, especially obesity, and also low weight are important elements in determining preterm delivery and should be considered in prenatal care.

Keywords: Obesity, maternal; Overweight; Premature birth; Pregnant woman.

\section{Resumen}

Introducción: El exceso de peso gestacional puede tener implicaciones para la salud de la gestante y del feto, y la prematuridad es un resultado importante, ya que a pesar de que las tecnologías en el cuidado neonatal están cada vez más avanzadas, el recién nacido prematuro puede necesitar complicaciones hospitalarias, ademáis de tener un mayor riesgo de muerte. Objetivo: Identificar y sintetizar la evidencia científica sobre la asociación entre obesidad durante el embarazo y prematuridad. Método: Se trata de una revisión de alcance, desarrollada en base a las recomendaciones de la guía internacional prisma-scr y al método propuesto por el Joanna Briggs Institute, Reviewers Manual 2017. Se realizó una búsqueda en las bases de datos electrónicas establecidas. Los datos fueron recolectados desde octubre/2020 hasta abril/2021. Resultados: Se recuperaron un total de 5.140 registros y se seleccionaron 42 estudios. De estos, $27(65,85 \%)$ presentaron una asociación estadísticamente significativa entre sobrepeso y prematuridad. El bajo peso de la mujer embarazada también se asoció con la prematuridad en el $27 \%$ de las investigaciones. Conclusión: Los cambios nutricionales en el embarazo, especialmente la obesidad, y también el bajo peso son elementos importantes en la determinación del parto prematuro y deben considerarse en la atención prenatal.

Palabras clave: Obesidad; Sobrepeso; Prematuridad; Embarazada.

\section{Introdução}

O Ganho de Peso Gestacional (GPG) está associado aos hábitos de vida pré-gestacionais, fatores genéticos, práticas alimentares, frequência de exercícios físicos, além da influência de opiniões de senso comum que afetam os aspectos psíquicos da gestante com suas inseguranças, anseios e medos sobre o gestar, incorrendo, muitas vezes, em contextos de vulnerabilidade alimentar (Dell"osbel et al., 2019). Os valores de referência instituídos para GPG pelo Institute of Medicine, dos Estados Unidos, é de 12,5 a $18 \mathrm{~kg}$ para mulheres com baixo peso, 11,5 a $16 \mathrm{~kg}$ em mulheres com Índice de Massa Corporal (IMC) normal, sete a $11 \mathrm{~kg}$ para gestantes que possuem sobrepeso e de cinco a $9 \mathrm{~kg}$ para as gestantes obesas (Ferreira et al., 2019). Este aumento de peso pode ser acompanhado através do IMC que estabelece os limites de acordo com a idade gestacional e classifica o estado nutricional da gestante em: baixo peso $\mathrm{IMC}<18,5$, peso adequado $18,5 \leq \mathrm{IMC} \leq 24,9$, sobrepeso $25,0 \leq$ IMC $\leq 29,9$ e obesidade IMC $\geq 30$.

O excesso de peso gestacional pode trazer implicações para a saúde da gestante e do concepto. Têm sido relatados desfechos perinatais negativos devido ao excesso de peso na gravidez, como cesárea, trabalho de parto prolongado ou laborioso, risco de pré-eclâmpsia, diabetes gestacional e prematuridade. Porém, há estudos que apresentam outro perfil de gestante que também possui desfechos negativos, com baixo peso pré gestacional e ganho de peso insuficiente de acordo com as recomendações, tendo maiores chances de partos prematuros em relação às obesas, trazendo uma falta de consenso acerca das implicações do excesso de peso gestacional em mulheres e recém-natos (Pedrine et al., 2020).

A prematuridade é um desfecho importante, pois apesar das tecnologias em assistência neonatal estarem cada vez mais avançadas, o recém-nascido prematuro pode necessitar de assistência hospitalar por complicações, além de ter maior risco de morte (Silva, 2019). As autoras da presente investigação realizaram uma revisão narrativa, assistemática, na Biblioteca Científica Eletrônica Online $(S c i E L O)$ e não identificaram revisões que sintetizassem estudos sobre excesso de peso e prematuridade, exceto, pela revisão de Nascimento et al (2018). Neste estudo, além de terem sido avaliadas bases de dados restritas, esta publicação buscou artigos de 1996 a 2016. Ambos os critérios de inclusão constituem possíveis limitações, sendo relevante a realização de nova revisão para ampliação da busca. Também não foi identificada nenhuma revisão sistemática acerca da pergunta do estudo na Biblioteca Cochrane. Assim, torna-se imperativa uma revisão sistematizada ampliando as bases de dados e sem limite temporal, sensível o suficiente para mapear a questão do excesso de peso gestacional e a prematuridade. $\mathrm{O}$ objetivo desta pesquisa consiste em mapear evidências na literatura sobre a associação entre o excesso de peso gestacional e a prematuridade. 


\section{Metodologia}

Trata-se de Scoping review, com protocolo de pesquisa registrado no Open Science Framework (https://osf.io/c7g94), desenvolvido com base nas recomendações do guia internacional PRISMA-ScR e no método proposto por Joanna Briggs Institute, Reviewers, Manual 2017 (Tricco et al., 2018). Este estabelece cinco etapas, sendo elas: 1) identificação da questão de pesquisa; 2) identificação dos estudos relevantes; 3) seleção dos estudos; 4) análise de dados; e, 5) agrupamento, síntese e apresentação dos dados.

A Scoping Review fornece um mapeamento dos principais conceitos que dão suporte a uma área de pesquisa, bem como esclarece as definições de trabalho e/ou as evidências científicas disponíveis para um determinado tópico (Tricco et al., 2018).

Elaborou -se um protocolo de pesquisa, onde constam as informações relativas à construção da pesquisa, por meio da estratégia participants, concept e context (PCC), em que P (participantes) são mulheres grávidas, C (conceito) é parto prematuro e C (contexto) refere-se ao excesso de peso e à obesidade. A questão de pesquisa definida foi: há evidências na literatura de que gestantes com excesso de peso são mais susceptíveis a apresentar parto prematuro?

Foram incluídas pesquisas com textos completos publicadas na íntegra em inglês, português e espanhol sobre parto prematuro em gestantes com obesidade, sem limite temporal. Foram excluídos editoriais, cartas, capítulos de livros e ensaios teóricos.

A pesquisa foi feita nas principais bases de dados: PubMed Central (PMC), Web of Science, Scopus e Science Direct e as que compõem o Portal Biblioteca Virtual em Saúde (BVS). A busca foi realizada entre setembro e outubro de 2020.

Inicialmente, realizou-se uma pesquisa dos descritores em ciências da saúde, a fim de identificar-se os descritores e palavras-chave mais frequentemente utilizadas nos estudos que abordavam a temática de interesse. Os termos MESH foram obtidos na National Library of Medicine - National Institutes of Health por meio do Medical Subject Heading, sendo, pregnant woman, obesity, maternal e premature birth. Em seguida, realizou-se a análise das publicações para identificar as palavraschave referentes a cada item da estratégia PCC. A estratégia geral adotada foi a busca pelos estudos utilizando os operadores booleanosand e or, sendo and entre os termos mesh e or entre os entretermos. Após a busca dos artigos, realizada com estratégias de busca adaptadas para cada base de dados, foi realizada a exportação dos resultados para o Software Rayyan, por meio do qual cinco pesquisadores, FASR, VJO, MVR, DBRS e ICT de forma independente, procederam à seleção dos artigos. As incongruências foram resolvidas em plenária, com a participação de uma sexta pesquisadora, MCCR.

Para a etapa de separação, sumarização e relatório dos elementos essenciais encontrados em cada estudo, foi utilizado um instrumento estruturado. Esse instrumento permitiu a síntese, interpretação dos dados e a análise numérica básica da extensão, natureza e distribuição dos estudos incorporados na revisão. Foram agrupados itens como, nome do artigo, autor, local do estudo, população, tipo de estudo, objetivos, instrumento de coleta de dados e principais resultados. Assim, em cada publicação foram identificados e extraídos os focos principais envolvidos na conjectura do problema, nos contextos, nos métodos, nas discussões e conclusões. Os artigos encontrados foram analisados, voltando-se aos textos integrais, sempre que necessário. Foi realizada uma análise temática do conteúdo dos artigos, visando mapear a literatura acerca do tema e verificar lacunas de pesquisa.

A síntese dos resultados foi apresentada na forma descritiva, por meio de quadros. Não houve necessidade de apreciação ética, uma vez que se trabalhou com dados de domínio público. Os níveis de evidência dos estudos foram classificados de acordo com a proposta do Joanna Briggs Institute, sendo categorizados de um a cinco e os graus de recomendação em A e B. Os níveis variam do maior A1 ao menor 4B (Institute, 2013). Os periódicos cujos artigos foram publicados foram analisados e classificados conforme o fator de impacto obtido no Journal Citation Reports (JCR). 


\section{Resultados}

Os artigos elegíveis nas bases de dados pesquisadas perfizeram um total de 5140 estudos, ficando 4822, após exclusão de 318 duplicatas. Após leitura de títulos e resumos e aplicando-se os critérios de inclusão e exclusão, foram excluídas 4733 publicações, restando 89 artigos. Foi então realizada a leitura dos artigos na íntegra e excluídos 47 deles, por não responderem à pergunta do estudo. Compuseram os resultados desta revisão 42 artigos (Figura 1). A síntese das informações gerais sobre os artigos está apresentada no Quadro 1.

Figura 1 - Fluxo referente ao processo de seleção dos estudos da Scoping Review, adaptado do PRISMA-ScR.

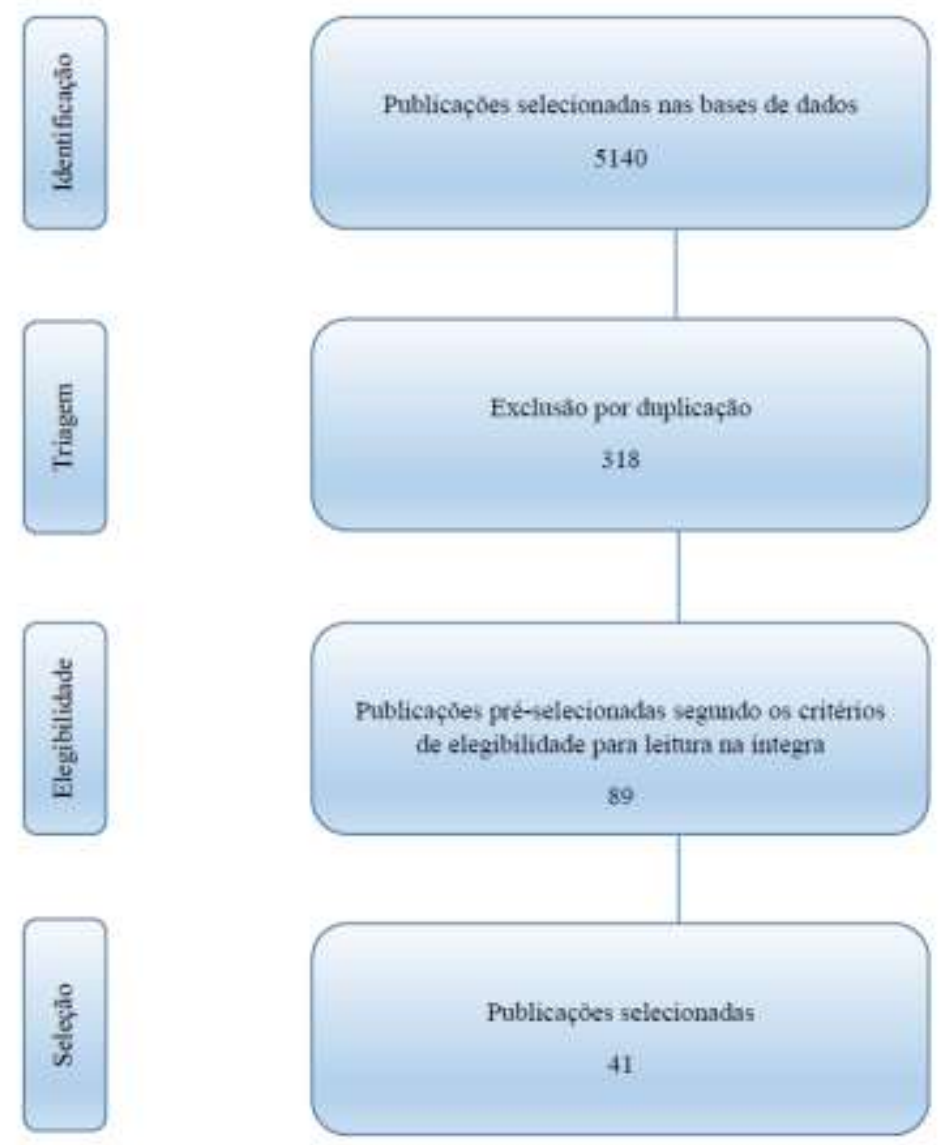

Fonte: Autores.

Quadro 1: Descrição dos estudos conforme objetivo e nível de evidência.

\begin{tabular}{|c|c|c|}
\hline Nome do artigo & Objetivo & NE* \\
\hline $\begin{array}{c}\text { Association of pre-pregnant body mass index and gestational } \\
\text { weight gain with timing of delivery }\end{array}$ & $\begin{array}{c}\text { Avaliar o efeito do índice de massa corporal pré-gravidez e do } \\
\text { ganho de peso gestacional sobre parto prematuro }\end{array}$ & 3B \\
\hline $\begin{array}{c}\text { Association among Maternal Obesity, Cervical Length, and } \\
\text { Preterm Birth }\end{array}$ & $\begin{array}{c}\text { Avaliar a associação entre o comprimento da cérvice no } 2^{\mathrm{a}} \\
\text { trimestre, o IMC materno e o parto pré-termo }\end{array}$ & 3B \\
\hline $\begin{array}{c}\text { Association of extremes of prepregnancy BMI with the clinical } \\
\text { presentations of preterm birth }\end{array}$ & $\begin{array}{c}\text { Elucidar a associação do IMC pré-gravidez com o ganho de } \\
\text { peso na ocorrência de parto prematuro e tamanho fetal }\end{array}$ & 3B \\
\hline Association of Maternal Obesity in Early Pregnancy with Adverse & Examinar as associações da obesidade materna no início da & 3B \\
\hline
\end{tabular}




\begin{tabular}{|c|c|c|}
\hline Pregnancy Outcomes: A Chinese Prospective Cohort Analysis & gravidez com resultados adversos da gravidez & \\
\hline $\begin{array}{l}\text { Association of Pre-Pregnancy Body Mass Index and Gestational } \\
\text { Weight Gain with Preterm Births and Fetal Size: an Observational } \\
\text { Study from Lebanon }\end{array}$ & $\begin{array}{l}\text { Elucidar a associação do IMC pré-gravidez com o ganho de } \\
\text { peso na ocorrência de parto prematuro e tamanho fetal }\end{array}$ & 3B \\
\hline $\begin{array}{l}\text { Body mass index and gestational weight gain as factors predicting } \\
\text { complications and pregnancy outcome }\end{array}$ & $\begin{array}{l}\text { Avaliar o impacto do índice de massa corporal (IMC) no início } \\
\text { da gestação e do ganho de peso no desfecho gestacional }\end{array}$ & 4B \\
\hline $\begin{array}{l}\text { Contribution of maternal overweight and obesity to the } \\
\text { occurrence of adverse pregnancy outcomes }\end{array}$ & $\begin{array}{c}\text { Avaliar a influência do sobrepeso e obesidade na gravidez e o } \\
\text { efeito benéfico das intervenções }\end{array}$ & 3B \\
\hline $\begin{array}{l}\text { Contribution of overweight and obesity to the occurrence of } \\
\text { adverse pregnancy outcomes in a multi-ethnic cohort: population } \\
\text { attributive fraction for Amsterdam }\end{array}$ & $\begin{array}{l}\text { Avaliar a influência da obesidade pré-gravidez e resultados } \\
\text { adversos }\end{array}$ & 3B \\
\hline $\begin{array}{l}\text { Effect of Body Mass Index on pregnancy outcomes in nulliparous } \\
\text { women delivering singleton babies }\end{array}$ & $\begin{array}{l}\text { Examinar a associação entre IMC e resultados perinatais em } \\
\text { nulíparas }\end{array}$ & 3B \\
\hline $\begin{array}{l}\text { Effect of maternal obesity on pregnancy outcomes in women } \\
\text { delivering singleton babies: a historical cohort study }\end{array}$ & $\begin{array}{l}\text { Examinar a associação entre IMC pré-gravidez e resultados } \\
\text { obstétricos e neonatais }\end{array}$ & 3B \\
\hline $\begin{array}{l}\text { Effects of Maternal Obesity on Antenatal, Perinatal, and Neonatal } \\
\text { Outcomes }\end{array}$ & Identificar complicações na gravidez devido à obesidade & 4B \\
\hline $\begin{array}{l}\text { Gestacional weight gain and pregnancy outcomes in relation to } \\
\text { Body Mass Index in asian indian women }\end{array}$ & Comparar ganho de peso gestacional e IMC & 3B \\
\hline $\begin{array}{l}\text { Gestational Weight Gain and Birth Outcome in Relation to } \\
\text { Prepregnancy Body Mass Index and Ethnicity }\end{array}$ & $\begin{array}{l}\text { Avaliar o ganho de peso na gravidez com indicadores relacionados } \\
\text { ao nascimento }\end{array}$ & 4B \\
\hline $\begin{array}{c}\text { Impact of Maternal Obesity on Perinatal Outcomes in Preterm } \\
\text { Prelabor Rupture of Membranes }>34 \text { Weeks }\end{array}$ & $\begin{array}{c}\text { Comparar os resultados da gravidez em mulheres obesas e não } \\
\text { obesas com RPM após } 34 \text { sem }\end{array}$ & 3B \\
\hline $\begin{array}{l}\text { Maternal and perinatal health outcomes by body mass index } \\
\text { category }\end{array}$ & $\begin{array}{l}\text { Avaliar o efeito do aumento da categoria de IMC materno e sua } \\
\text { relação com resultados de saúde materna e perinatal }\end{array}$ & 3B \\
\hline $\begin{array}{l}\text { Maternal BMI and preterm birth: A systematic review of the } \\
\text { literature with meta-analysis }\end{array}$ & $\begin{array}{c}\text { Examinar a associação entre alto índice de massa corporal materna } \\
\text { (IMC) na pré-gravidez e o risco de parto prematuro (PTB) }\end{array}$ & $3 \mathrm{~A}$ \\
\hline Maternal obesity, associated complications and risk of prematurity & $\begin{array}{c}\text { Examinar as taxas de obesidade ao longo de } 12 \text { anos. Determinar a } \\
\text { influência da raça na associação entre obesidade e IG do RN. } \\
\text { Estudar os efeitos da obesidade e obesidade mórbida na gestação }\end{array}$ & 3B \\
\hline Maternal obesity, gestational hypertension, and preterm delivery & $\begin{array}{l}\text { Estudar a obesidade materna como fator de risco para parto } \\
\text { prematuro }\end{array}$ & 3B \\
\hline $\begin{array}{l}\text { Maternal pregnancy body mass index, gestacional weight gain, and } \\
\text { risk of adverse perinatal outcomes in Taiwan: a population-based } \\
\text { birth cohort study }\end{array}$ & $\begin{array}{c}\text { Avaliar a associação do IMC pré-gestacional, ganho de peso } \\
\text { gestacional e risco de resultados perinatais adversos }\end{array}$ & $3 \mathrm{~A}$ \\
\hline $\begin{array}{c}\text { Obesity as a Predictor of Adverse Maternal Outcomes among } \\
\text { Pregnant Women of a rural community }\end{array}$ & $\begin{array}{l}\text { Determinar os resultados maternos da gravidez entre mulheres } \\
\text { com aumento do IMC }\end{array}$ & 3B \\
\hline $\begin{array}{l}\text { Outcomes of Gestational Weight Gain Outside the Institute of } \\
\text { Medicine Guidelines }\end{array}$ & $\begin{array}{l}\text { Comparar os resultados da gravidez entre mulheres que deram à } \\
\text { luz no Hospital Lerdsin com ganho de peso gestacional (GWG) } \\
\text { dentro e acima ou abaixo das diretrizes do Institute of Medicine } \\
\text { (IOM) }\end{array}$ & 3B \\
\hline $\begin{array}{l}\text { Pre-pregnancy BMI and weight gain: where is the } \\
\text { tipping point for preterm birth? }\end{array}$ & $\begin{array}{c}\text { Examinar o efeito do IMC pré-gravidez materno e ganho de peso } \\
\text { durante a gravidez no nascimento PT }\end{array}$ & 3B \\
\hline Pre-pregnancy maternal obesity in Greece: A case-control analysis & $\begin{array}{l}\text { Investigar a associação entre o índice de massa corporal pré- } \\
\text { gravidez e o risco de parto cesáreo, internação em Unidade de } \\
\text { Terapia Intensiva Neonatal, macrossomia e parto prematuro }\end{array}$ & $2 \mathrm{D}$ \\
\hline Pre-pregnancy obesity compromises obstetric and neonatal & Avaliar se obesidade pode afetar resultados obstétricos e neonatais & 3B \\
\hline
\end{tabular}




\begin{tabular}{|c|c|c|}
\hline outcomes & & \\
\hline $\begin{array}{l}\text { Preconceptional and maternal obesity: epidemiology and health } \\
\text { consequences }\end{array}$ & $\begin{array}{c}\text { Descrever as tendências no mundo e a prevalência da obesidade } \\
\text { entre mulheres especialmente aquelas em países menos capazes de } \\
\text { promover cuidados em saúde }\end{array}$ & $2 \mathrm{~B}$ \\
\hline Pregnancy in obese women & $\begin{array}{c}\text { Estudar gravidez e suas complicações em mulheres obesas usando } \\
\text { um projeto de controle de caso }\end{array}$ & 4B \\
\hline $\begin{array}{c}\text { Prepregnancy Body Mass Index and Pregnancy Weight Gain: } \\
\text { Associations With Preterm Delivery }\end{array}$ & $\begin{array}{l}\text { Examinar a associação entre ganho de peso na gravidez e o parto } \\
\text { pretermo entre mulheres com diferentes índices de IMC }\end{array}$ & 4B \\
\hline $\begin{array}{l}\text { Prepregnancy body mass index, gestacional weight gain, and } \\
\text { pregnancy outcomes in China }\end{array}$ & Analisar obesidade materna e seus efeitos neonatais & 3B \\
\hline $\begin{array}{c}\text { Prepregnancy Body Size, Gestational Weight Gain, and Risk of } \\
\text { Preterm Birth in African-American Women }\end{array}$ & Investigar a relação entre o nascimento prematuro e o IMC & $3 \mathrm{~B}$ \\
\hline $\begin{array}{l}\text { Prepregnancy Overweight and Obesity Are Associated with an } \\
\text { Increased Risk of Preterm Birth in Chinese Women. }\end{array}$ & $\begin{array}{l}\text { Estudar a associação entre o índice de massa corporal inicial } \\
\text { (IMC) e risco de parto prematuro em mulheres chinesas }\end{array}$ & 3B \\
\hline $\begin{array}{c}\text { Rate of gestational weight gain, pre-pregnancy body mass index and } \\
\text { preterm birth subtypes: a retrospective cohort study from Peru }\end{array}$ & $\begin{array}{c}\text { Examinar a associação entre a taxa de ganho de peso gestacional, } \\
\text { IMC pré-gestacional e o nascimento pré-termo }\end{array}$ & 3B \\
\hline $\begin{array}{c}\text { Relationships between pregnancy outcomes, biochemical markers } \\
\text { and pre-pregnancy body mass index }\end{array}$ & $\begin{array}{c}\text { Analisar a relação entre os resultados da gravidez, os marcadores } \\
\text { bioquímicos e o índice de massa corporal pré-gravidez }\end{array}$ & 4B \\
\hline $\begin{array}{l}\text { Risks associated with obesity in pregnancy, for the mother and } \\
\text { baby: a systematic review of reviews }\end{array}$ & $\begin{array}{c}\text { Obter uma visão geral dos riscos relacionados à obesidade em } \\
\text { mulheres grávidas }\end{array}$ & $3 \mathrm{~A}$ \\
\hline $\begin{array}{l}\text { Risks of pregnancy and birth in obese primiparous women: an } \\
\text { analysis of German perinatal statistics. }\end{array}$ & $\begin{array}{l}\text { Comparar os riscos de gravidez e parto em obesas e mulheres de } \\
\text { peso normal dando à luz seu primeiro filho }\end{array}$ & 3B \\
\hline Severe obesity, gestational weight gain, and adverse birth outcomes & $\begin{array}{c}\text { Explorar a associação entre GPG e nascimentos pequenos para IG, } \\
\text { GIG, AIG e prematuros }\end{array}$ & 4B \\
\hline $\begin{array}{l}\text { Spontaneous and indicated preterm delivery ris kis increased among } \\
\text { overweight and obese women without prepregnancy chronic disease }\end{array}$ & $\begin{array}{l}\text { Investigar o risco de parto prematuro associado com o IMC pré- } \\
\text { gravidez }\end{array}$ & 3B \\
\hline $\begin{array}{l}\text { The Association Between Prepregnancy Maternal Body Mass Index } \\
\text { and Preterm Delivery }\end{array}$ & $\begin{array}{c}\text { Investigar a associação entre o IMC materno pré-gestacional com } \\
\text { o parto pré-termo menos de } 37 \text { sem e menos que } 34 \text { sem }\end{array}$ & $2 \mathrm{D}$ \\
\hline The effect of body mass index on delivery outcomes & $\begin{array}{l}\text { Encontrar a relação entre pré-gravidez, índice de massa corporal } \\
\text { materno (IMC), parto prematuro espontâneo e peso ao nascer }\end{array}$ & 3B \\
\hline $\begin{array}{l}\text { The effect of body mass index value during labor on pregnancy } \\
\text { outcomes in Turkish population (obesity and pregnancy outcomes) }\end{array}$ & $\begin{array}{c}\text { Investigar a relação do IMC com os resultados de parto e gravidez } \\
\text { em mulheres turcas }\end{array}$ & 4B \\
\hline $\begin{array}{l}\text { The effects of morbid obesity on maternal and neonatal health } \\
\text { outcomes: a systematic review and meta-analyses. }\end{array}$ & $\begin{array}{l}\text { Compreender os efeitos da obesidade mórbida nas mães e } \\
\text { resultados de saúde neonatal }\end{array}$ & 3B \\
\hline $\begin{array}{c}\text { The Impact of Maternal Obesity and Excessive Gestational Weight } \\
\text { Gain on Maternal and Infant Outcomes in Maine: Analysis of } \\
\text { Pregnancy Risk Assessment Monitoring System Results from } 2000 \\
\text { to } 2010\end{array}$ & $\begin{array}{c}\text { Compreender as relações entre a obesidade pré-gestacional, o } \\
\text { ganho de peso gestacional excessivo e desfechos maternos e fetais } \\
\text { adversos }\end{array}$ & $4 B$ \\
\hline $\begin{array}{c}\text { Trends in Smoking and Overweight During Pregnancy: Prevalence, } \\
\text { Risks of Pregnancy Complications, and Adverse Pregnancy } \\
\text { Outcomes }\end{array}$ & $\begin{array}{l}\text { Descrever tendências na prevalência de tabagismo e sobrepeso, e } \\
\text { avaliar a associação e riscos relacionados à gravidez associada. }\end{array}$ & 2B \\
\hline
\end{tabular}

*NE: Nível de evidência científica. Fonte: Autores. 
Ao analisar os dados obtidos, identificou-se que a primeira publicação sobre associação de obesidade e prematuridade ocorreu em 1993 (Konje et al., 2016) e a próxima publicação foi registrada somente sete anos após, no ano 2000 (Schieve et al., 2000). Os anos que tiveram mais estudos publicados foram 2010 (Aly et al., 2010; Briese et al., 2011; Madan et al., 2010; Wise et al., 2010; Zhong et al., 2010) e 2016 (Baugh et al., 2016; Palatnik et al., 2016; Papachatzi et al., 2016; Poston \& Rishi, 2016; Rafei et al., 2016) com o total de cinco estudos $(11,9 \%)$. Foi identificado também que $21,4 \%$ das publicações aconteceram nos últimos cinco anos, sendo as mais recentes em 2020 (Chen et al., 2020; Su et al., 2020).

Evidenciou-se que a maioria dos estudos são internacionais, os locais com mais estudos foram o continente americano responsável por 52,3\% das publicações, seguido por 26,1\% na Ásia, 19,0\% na Europa, e 4,7\% na Oceania.

Foi obtido o fator de impacto dos periódicos cujos artigos desta investigação foram publicados. Este variou entre o menor fator de 0,046 (Rafei et al., 2016) e o maior encontrado 25,340 (Chen et al., 2010).

A análise da associação entre excesso de peso na gravidez e prematuridade mostrou três conjuntos de resultados. O primeiro conjunto mostra as investigações que encontraram associação positiva entre excesso de peso gestacional e parto prematuro (Avci et al., 2014; Bhattacharya et al., 2007; Bhavadharini et al., 2017; Bodnar et al., 2010; Carnero et al., 2012; Chen et al., 2020; Cnattingius \& Lambe, 2002; Djelantik et al., 2011; Dodd et al., 2011; Han et al., 2011; Hancke et al., 2015; Kim et al., 2017; Lutsiv et al., 2015; Marchi et al., 2015; Masho et al., 2013; Madan et al., 2010; Papachatzi et al., 2016; Parveen et al., 2017; Poston \& Rishi, 2016; Rafei et al., 2016; Savitz et al., 2011; Su et al., 2020; Torloni et al., 2010; Wise et al., 2010; Yang et al., 2019; Zhong et al., 2010; Zhou et al., 2019) perfazendo um total de 64,2\% dos estudos. O segundo grupo são artigos que não encontraram associação significativa entre essas variáveis, em um total de nove publicações (Aly et al., 2010; Aydin et al., 2010; Briese et al., 2011; Chen et al., 2010; Gonçalves et al., 2012; Lynch et al., 2019; Melchor et al., 2019; Palatnik et al., 2016; Puscasi et al., 2013). E o último grupo são de pesquisas que evidenciaram associação negativa entre excesso de peso na gravidez e prematuridade com um percentual de 14,2\% (Asvanarunat, 2014; Jenabi \& AslToghiri, 2011; Konje et al., 2016; Lynch et al., 2014; Schieve et al., 2000).

\subsection{Associação positiva entre excesso de peso na gravidez e prematuridade}

A maioria dos estudos evidenciou a associação positiva entre excesso de peso gestacional com o desfecho parto prematuro. Foram descritas variáveis associadas a este aumento de peso, estas, por sua vez, expõem o feto ao risco do desfecho em questão, além de complicações para a gestante. É possível elucidar as evidências destes trabalhos através do tipo de metodologia utilizada.

Os estudos de coorte evidenciam que o IMC materno é um fator de risco para o parto prematuro induzido e espontâneo, parto cesárea, ruptura prematura de membranas e nascimento de bebês Grande para a Idade Gestacional (GIG). Um estudo realizado em Aberdeen, na Escócia, evidenciou que o parto prematuro antes de 33 semanas aumentou significativamente em mulheres com peso mais elevado (Bhattacharya et al., 2007). Outra coorte realizada em Boston e Massachusetts procurou o efeito do IMC e do ganho de peso durante a gravidez no parto prematuro e evidenciou que a taxa de parto prematuro espontâneo com ruptura prematura de membranas entre as mulheres com sobrepeso diminuiu com a redução do ganho de peso gestacional e inversamente aumentou entre as mulheres que tiveram ganho de peso gestacional excessivo (Masho et al., 2013).

Uma coorte realizada em Pittsburgh, nos Estados Unidos, mostrou que bebês de mães com obesidade tiveram desfechos graves nos resultados da gravidez, como parto pré-termo, natimorto e maior realização do parto cesárea, além da mortalidade materna. Sugeriu também que dependendo do ganho de peso na gravidez e o nível de obesidade envolvido, pode haver uma maior exposição aos riscos mencionados (Bodnar et al., 2010). 
Dois estudos de revisão indicaram maior risco de cesárea, admissão em UTI neonatal e prematuridade, assim como, mais chance de abortamento, malformação fetal, bebês GIG, distorcia de ombro e natimortos. Considerou-se também mais chances de parto prematuro por indicação médica (Poston \& Rishi, 2016; Torloni et al., 2010).

Outro estudo de análise transversal na Coreia evidenciou que a população com obesidade tem menor escolaridade e menor renda quando comparada à população eutrófica. Adicionalmente, concluiu-se que os resultados gestacionais também sofreram influência do excesso de peso, como aumento do nível de estresse, homocisteína no plasma sanguíneo, parto prematuro, maior probabilidade de desenvolver comorbidade como pré-eclâmpsia, restrição do crescimento intrauterino (CIUR) e diabetes gestacional (Han et al., 2011).

\subsection{Ausência de associação entre excesso de peso na gravidez e prematuridade}

Um estudo do tipo transversal, realizado em 2010, aponta que o excesso de peso ou obesidade não estão relacionados com o início de trabalho de parto pré-termo espontâneo, provavelmente, devido ao colo destas mulheres se apresentarem mais longo. Porém, foi constatado um aumento significativo na ruptura prematura das membranas, o que expõe ao nascimento prematuro, mas não necessariamente, evoluindo para o parto (Zhong et al., 2010).

Uma coorte que realizou um acompanhamento ao longo de 12 anos nos EUA, identificou um risco aumentado de parto prematuro em mulheres com obesidade mórbida, na análise bruta, no entanto, ao efetivar a análise multivariada, o excesso de peso não se manteve no modelo (Aly et al., 2010).

Autores analisaram o IMC e o ganho de peso ponderal com os resultados de parto e nascimento e encontraram risco de prematuridade associado à magreza extrema (Lynch et al., 2014), um estudo de coorte histórica, que examinou a relação entre IMC pré gestacional e resultados adversos na gestação não encontrou associação entre obesidade e prematuridade (Melchor et al., 2019). Outro estudo não encontrou essa associação, apesar de identificar associação com desfechos desfavoráveis, como sepse neonatal, complicações respiratórias e necessidades de terapias medicamentosas (Lynch et al., 2019).

Um trabalho transversal no Brasil, com 1.117 puérperas, não identificou relação entre IMC materno e parto pré-termo, porém traz que quanto maior o IMC e o ganho de peso gestacional, maior o risco de parto cirúrgico (Gonçalves et al., 2012).

\subsection{Associação negativa entre excesso de peso na gravidez e prematuridade}

Dentre os estudos que encontraram associação negativa entre excesso de peso gestacional e prematuridade, destacamse dois trabalhos realizados, sendo um nos Estados Unidos e outro no Irã. Esses estudos de coorte evidenciaram que mulheres que possuíam um baixo peso pré-gestacional e que permaneceram com baixo ganho de peso durante a gestação, tiveram um IMC baixo e consequentemente, uma maior frequência de parto prematuro em relação às outras mulheres. A amostra somada constituiu 18.496 binômios mãe-filho, e ainda que os locais destes estudos estejam situados em continentes com culturas distintas e opostas, os resultados obtidos foram semelhantes (Palatnik et al., 2016; Jenabia \& AslToghiria, 2011).

Estudo de caso-controle com 750 mulheres, realizado entre o ano 1988 e 1990 no Reino Unido, buscou identificar as complicações em gestantes obesas, este por sua vez encontrou que estas mulheres estão menos expostas ao risco de parto prematuro, tornando a obesidade um fator de proteção para o desfecho prematuridade (Konje et al., 2016). Entre outubro de 2010 e setembro de 2013 foi realizado um estudo na Tailândia com 3683 mulheres, evidenciando menor risco de prematuridade na gestação com maior ganho de peso (Asvanarunat, 2014).

Utilizando os dados de um estudo nacional no ano de 1988, nos Estados Unidos, os autores de um estudo do tipo quantitativo coletaram informações de prontuários médicos. Essa investigação obteve dados de ganho de peso gestacional e, 
dentre os partos prematuros ocorridos naquele ano, concluiu-se que mulheres com baixo peso na gravidez foram as que mais frequentemente tiveram bebês prematuros (Schieve et al., 2000).

\section{Discussão}

Os resultados da presente investigação mostram que não há um consenso na associação entre excesso de peso e prematuridade. Uma possível justificativa para a falta de consenso entre os estudos refere-se às comorbidades associadas ao excesso de peso. Este é fator predisponente para o diabetes gestacional e, portanto, também pode contribuir para este desfecho. $\mathrm{O}$ aumento da resistência à insulina sobrecarrega o feto, com uma maior passagem de glicose via placentária, acarretando em riscos para a saúde materna, como o desenvolvimento da DM tipo II no pós parto, além de expor o bebê a uma maior probabilidade de parto prematuro. Em estudo recente realizado com prematuros, das comorbidades mais desenvolvidas por suas mães, a pré-eclâmpsia e a diabetes gestacional foram as mais frequentes (Adams et al., 2021).

Acredita-se que há também outros fatores envolvidos que podem ter contribuído para a prematuridade, como a Doença Hipertensiva Específica da Gravidez (DHEG), que por questões metabólicas, é muito comum em gestantes obesas. É observado na literatura que esta comorbidade materna afeta o prognóstico neonatal, ao avaliar gestantes diagnosticadas com pré-eclâmpsia, apresentando desfechos perinatais desfavoráveis, principalmente a alta prevalência de internações neonatais em UTI (Manera \& Höfelmann, 2017; Sousa et al., 2020).

Adicionalmente, na presente revisão, houve estudos que não encontraram associação entre excesso de peso na gestação e prematuridade. Uma possível justificativa é, talvez, a presença de outros fatores que sabidamente se relacionam com o parto prematuro, como idade, má assistência no pré-natal e baixa renda, em detrimento com o excesso de peso isoladamente (Otaviano et al., 2021). Destaca-se que uma das maiores dificuldades da obstetrícia que determina altas taxas nos partos prematuros e abortamentos é a Incompetência Istmo Cervical (IIC). Somente esta condição representa um fator de risco, porém, se associada com outros fatores, pode complicar a evolução da gestação e sua vitalidade. Outro fator importante é a assistência pré-natal inadequada, o que contribui para um acompanhamento ineficaz, incorrendo em desfechos que poderiam ter sido evitados (Soares et al., 2020).

Uma investigação mostra a magreza como um fator causador de parto prematuro, apresentando associação negativa na combinação obesidade e prematuridade. Determinadas alterações nutricionais envolvidas, dificuldade no acesso a alimentos fonte de boas calorias devido ao baixo poder socioeconômico, se consolidam como fatores associados ao baixo peso gestacional e ganho de peso inadequado. É importante que a gestante possua uma boa ingestão de micronutrientes em sua dieta para que, além de suprir a necessidade nutricional que a gestação exige, possa manter as condições favoráveis para o desenvolvimento pleno do feto. A gestação é um período de mudanças fisiológicas na mulher, mudando as necessidades nutricionais e a alimentação. Os nutrientes são fundamentais durante a gestação, pois garantem a evolução esperada do feto. Os micronutrientes compõem vitaminas e minerais necessários para a interação entre o compartimento fetal, placentário e materno (Gonçalves et al., 2019).

Dentre os micronutrientes necessários para o crescimento fetal, salienta-se o ácido fólico e o ferro. O ácido fólico é essencial para ampliar o número de células, para o crescimento da placenta e do embrião, além da prevenção de anemias. A carência desse micronutriente pode levar a deformações no tubo neural e espinha bífida (Ferraz et al., 2018).

A orientação para que a gestante tenha uma alimentação saudável é a mesma da população geral, dieta variada, colorida, harmoniosa e segura, baseada em alimentos in natura, como frutas e verduras, cereais integrais, carnes brancas como peixes e frango sem pele, carnes vermelhas magras, porém algumas adaptações são necessárias visando garantir o suprimento de macro e micronutrientes tanto para a mãe como para o feto (Gonçalves et al., 2019).

Uma investigação mostra a magreza como um fator causador de parto prematuro, apresentando associação negativa na 
combinação obesidade e prematuridade. Determinadas alterações nutricionais envolvidas, dificuldade no acesso a alimentos fonte de boas calorias devido ao baixo poder socioeconômico, se consolidam como fatores associados ao baixo peso gestacional e ganho de peso inadequado. É importante que a gestante possua uma boa ingestão de micronutrientes em sua dieta para que, além de suprir a necessidade nutricional que a gestação exige, possa manter as condições favoráveis para o desenvolvimento pleno do feto. Essa questão pode, talvez, justificar o fato de bebês de mulheres com IMC baixo ou inadequado terem baixo peso ao nascer, serem pequenos para a idade gestacional e, muitas vezes, nascerem prematuramente (Oliveira et al., 2018).

Nesta revisão há diversos estudos internacionais, mostrando diferentes realidades para um mesmo desfecho, porém, é válido salientar que há raros estudos brasileiros sobre o assunto, demonstrando a importância desta revisão, pois os fatores de obesidade e prematuridade podem mudar de uma etnia para outra (Souza et al., 2018).

Também é possível observar que, a maior parte dos estudos são de coorte, ou seja, nível 3B de evidência, apontando uma qualidade razoável das investigações e mostrando evidências consistentes acerca da pergunta de pesquisa (Camargo et al., 2019).

A presente revisão de escopo incluiu, em seus achados, um artigo de revisão sistemática, realizado e publicado por autores brasileiros no ano de 2009. Diferentemente de nossa investigação, esta revisão sistemática limitou o período de busca, sendo de janeiro de 1968 a janeiro de 2008 e pesquisou as bases de dados Embase, Cinahl, Lilacs e Medline. Corroborando nossos achados, a maior parte dos estudos mostrou que houve associação entre obesidade gestacional e prematuridade (Torloni et al., 2010).

Este estudo encontrou taxas altas de sobrepeso e obesidade em países desenvolvidos, em uma revisão sistemática, verificou a prevalência de obesidade materna em diversos países e identificou que os países de renda baixa e média vivenciam grandes mudanças nos padrões de dieta e atividade física durante as últimas três décadas em um processo denominado transição nutricional, onde o consumo de alimentos com alto teor de açúcar e gordura e pobre em nutrientes, são maiores, além disso o estilo de vida moderna resulta em sedentarismo, isso justifica taxas elevadas em regiões mais avançadas economicamente como Chicago e Taiwan (Poston et al., 2016).

Segundo o VIGITEL, um levantamento feito pelo Ministério da Saúde via telefone em 2009, 42,3\% das mulheres participantes estavam acima do peso. Já de acordo com a Pesquisa Nacional de Saúde (PNS) divulgada em 2019, 62,6\% das mulheres estão acima do peso (IBGE, 2019). Esses achados reforçam a importância de pesquisas sobre o tema com gestantes brasileiras.

Faz-se importante salientar que, nesta revisão de escopo, a prematuridade foi um achado relevante nos estudos incluídos. Sabe-se que a assistência ao recém-nascido prematuro tem se aprimorado com o passar dos anos, com avanços tecnológicos e necessidade, cada vez mais frequente, de atualização dos recursos humanos envolvidos. Entretanto, esse preparo e evolução da assistência consequentemente trazem custos operacionais maiores, pois estes bebês, muitas vezes, demandam da assistência em unidades de cuidados intensivos, além de maiores necessidades assistenciais especializadas à família (Baseggio et al., 2017).

A prematuridade traz a possibilidade de problemas como insuficiência respiratória, lesões no cérebro e deficiência neurológica entre outros e consequentemente a internação (Rodrigues, 2018). A hospitalização deste recém-nascido tende a repercutir de maneira negativa, podendo prejudicar o vínculo do binômio mãe e filho, desencadeando consequências no contexto familiar, visto que, essa família pode ter outros filhos, ausência de rede de apoio e várias outras situações que demandam de uma rotina já estabelecida. Além disso, a chegada precoce do bebê pode anular as expectativas relacionadas ao nascimento do bebê (Baseggio et al., 2017).

O puerpério da mãe de um bebê prematuro se torna uma jornada de aprendizado ainda maior e cheia de desafios. 
Ademais, existem evidências reforçando que a permanência do bebê no hospital pode ser gatilho de diversos transtornos afetivos na mãe, principalmente transtornos mentais específicos do puerpério, uma vez que essa mulher pode se sentir aflita e ameaçada em sua função de mãe (Azzi, 2018).

Baseggio et al (2017) apontam a prematuridade como uma das condições que mais prejudicam o desenvolvimento do recém-nascido. A prematuridade pode implicar de forma negativa no desenvolvimento motor, no desenvolvimento social, no desempenho intelectual e na linguagem desse indivíduo em idades posteriores (Lima et al., 2019). Com isto, é necessário todo um preparo da família para receber este bebê. Se preparar com antecedência e envolver a família no cuidado auxilia no desenvolvimento do bebê e compreende a individualidade da família, o que promove uma sensação de aproximação dos pais. (Baseggio et al., 2017).

Outro ponto crucial na prematuridade é a promoção do aleitamento materno. Estão consolidados na literatura os benefícios da amamentação para mães e bebês, em especial, os prematuros. Nesse público, a amamentação reduz a incidência e as complicações de enterocolite necrosante, sepse e retinopatia da prematuridade, promove o desempenho neuropsicomotor, fortalecimento do vínculo mãe-filho, um período reduzido de hospitalização e também modera o número de reinternações. As taxas de aleitamento materno neste grupo, no entanto, ainda estão abaixo do recomendado pela Organização Mundial de Saúde (OMS) e isso se deve em grande parte pelo desmame mesmo após o estabelecimento do aleitamento materno no ambiente hospitalar (Lima et al., 2019). Durante a internação hospitalar tem-se barreiras como transporte, armazenamento, vulnerabilidade e até mesmo disponibilidade desta mãe para amamentar.

Outro aspecto relevante nesse estudo, é a constatação de que o excesso de peso na gestação está em elevação, demandando cuidados específicos no contexto da Atenção Primária à Saúde (Almeida et al., 2017). De fato, tem-se acompanhado um aumento alarmante do sobrepeso e da obesidade ao longo dos anos, sendo que as mulheres, compõem o maior grupo da população com esse agravo (Souza et al., 2019).

Apresenta-se uma necessidade ainda maior de prevenção do excesso de peso em mulheres. Essa prevenção pode envolver aconselhamento, incentivo à atividade física e a prática de hábitos saudáveis, incluindo alimentação e também a realização de atividades que visam a promoção da saúde, como já mencionado na literatura e em consonância com as recomendações da OMS (Almeida et al., 2017). Outro aspecto preocupante do excesso de peso é que ele é uma conhecida razão para o desprezo social e, sobretudo, observado em uma sociedade onde o ideal proposto pela mídia é de um corpo magro e escultural. A percepção corporal no ciclo gravídico tende a ser satisfatória para a gestante, que vê as mudanças no seu corpo em razão da espera do seu bebê, contudo, a tensão da gestante com sobrepeso em relação a permanência do excesso de peso após a gestação é aparente, tensão essa que parte do estigma social em relação a esta condição. Não aceitar seu corpo pode ser motivo até mesmo, para redução da oferta do aleitamento materno. $\mathrm{O}$ excesso de peso, as alterações na forma e no tamanho corporal podem desatar nessa mulher uma responsabilização por tal fato e desvalorização na autoestima, minimizando o autocuidado (Pires et al., 2018).

Esse estudo apresenta limitações. Uma delas é o fato de esta revisão não abranger todas as bases de dados existentes, desta forma, podendo haver estudos que respondam à pergunta desta pesquisa e não tenham sido incluídos. Esta revisão mapeou, de forma sistematizada, os estudos acerca da associação entre excesso de peso gestacional e prematuridade, contribuindo com a literatura nesse campo de investigação.

Encontra-se a necessidade de entender o universo da associação obesidade materna e prematuridade no âmbito nacional, buscando uma maior produção científica, uma vez que os estudos de autores brasileiros encontrados em nossa pesquisa foram insuficientes em relação aos estudos internacionais. 


\section{Conclusão}

A presente revisão de escopo não obteve consenso sobre a associação entre excesso de peso gestacional e prematuridade. No entanto, faz-se oportuno considerar que a maioria dos autores aponta que a obesidade está associada não só com a prematuridade, mas também com outros diversos resultados maternos e/ou neonatais, como por exemplo, diabetes gestacional, distócias, bebês GIG, abortamento, pré-eclâmpsia, sepse neonatal e terapias medicamentosas. Outro ponto relevante é que a magreza também se apresentou como fator predisponente para prematuridade.

Além disso, o mapeamento das evidências acerca do tema, mostrou que a maior parte dos estudos são internacionais, por tanto os autores deste artigo sugerem que são necessárias investigações com amostras de gestantes brasileiras, especialmente os estudos longitudinais, sobre impactos do excesso de peso nas taxas de prematuridade, bem como outros fatores que alteram os resultados perinatais.

\section{Referências}

Adams, F. C., Gomes, J. S., Strassburger, M. J., Ferreira, P. F., Colet, C. d., \& Strassburger, S. Z. (2021). Factors associated to the gestational age of prematuros inhibited in a neonatal intensive therapy unit. R. pesq.: cuid. fundam. online, 13, 158-163.

Almeida, L., Campos, k., Randow, R., \& Guerra, V. (2017). Estratégias e desafios da gestão da Atenção Primária à Saúde no controle e prevenção da obesidade. Revista Eletronica Gestão \& Saúde, 8(1).

Aly, H., Hammad, T., A. N., Mohamed, M., Bathgate, S., \& El-Mohandes, A. (2010). Maternal obesity, associated complications and risk of. Journal of Perinatology, 30(7), 447-451.

Asvanarunat, E. (2014). Outcomes of gestational weight gain outside the Institute of Medicine Guidelines. Journal of the Medical Association of Thailand = Chotmaihet thangphaet, 97(11), 1119-1125.

Avcı, M. E., Şanlıkan, F., Çelik, M., Avcı, A., Kocaer, M., \& Göçmen, A. (2014). Effects of Maternal Obesity on Antenatal, Perinatal, and Neonatal. Journal of Maternal-Fetal \& Neonatal Medicine, 28(17), 1-12.

Aydin, C., Baloglu, A., Yavuzcan, A., \& Inci, A. (2010). The effect of body mass index value during labor on pregnancy outcomes in Turkish population (obesity and pregnancy outcomes). Arch Gynecol Obstet, 281(1), 49-54.

Azzi, D. (2018). Depressão pós-parto e desenvolvimento infantil nos três primeiros anos de vida. Dissertação de Mestrado. Programa de Pós graduação em Psicologia da Saúde da Universidade Metodista de São Paulo, 1-94.

Baseggio, D. B., Dias, M. P., Brusque, S. R., Donelli, T. M., \& Mendes, P. (2017). Vivências de mães e bebês prematuros durante a internação neonatal. Temas psicol, 25(1), 154-167.

Baugh, N., Harris, D. E., Aboueissa, A.-M., Sarton, C., \& Lichter, E. (2016). The Impact of Maternal Obesity and Excessive Gestational Weight Gain on Maternal and Infant Outcomes in Maine: Analysis of Pregnancy Risk Assessment Monitoring System Results from 2000 to 2010. Journal of Pregnancy, 1-10.

Bhattacharya, S., Campbell, D. M., Liston, W. A., \& Bhattacharya, S. (2007). Effect of Body Mass Index on pregnancy outcomes in nulliparous women delivering singleton babies. BMC Public Health, 7(1), 1-8.

Bhavadharini, B., Anjana, R. M., Deepa, M., Jayashree, G., Nrutya, S., Malanda, M. S., . . . Mohan, V. (2017). Gestational weight gain and pregnancy outcomes in relation to body mass index in Asian Indian women. Indian Journal of Endocrinology and Metabolism, 21(4), 588-593.

Bodnar, L. M., Siega-Riz, A. M., Simhan, H. N., Himes, K. P., \& Abrams, B. (2010). Severe obesity, gestational weight gain, and adverse birth outcomes. American Journal of Clinical Nutrition, 91(6), 1642-1648.

Briese, V., Voigt, M., Wisser, J., Borchardt, U., \& Straube, S. (2011). Risks of pregnancy and birth in obese primiparous women: an analysis of german perinatal statistics . Arch Gynecol Obstet, 283(2), 249-253.

Bodnar, L.M., Siega-Riz, A.M., Simhan, H.N., Himes, K.P., \& Abrams, B. (2010). Severe obesity, gestacional weight gain, and asverse birth outcomes. American Journal of Clinical Nutrition, 91(6), 1642-1648.

Camargo, L. M., Silva, R. P., \& Meneguetti, D. U. (2019). Research methodology topics: Cohort studies or prospective and retrospective cohort studies. J. Hum. Growth Dev., 29(3), 1-4.

carmona, A. P. (Dezembro de 2020). Prescrição de exercicos fisicos nas grávidas obesas e com sobrepeso e os seus impactos nos outcomes cardiorespiratórios: Uma revisão sistemática. P.Porto.

Carnero, A., Mejía, C., \& García, P. (2012). Rate of gestational weight gain, pre-pregnancy body mass index and preterm birth subtypes: A retrospective cohort study from Peru. BJOG An International Journal of Obstetrics and Gynaecology, 119(8), 924-935. 
Chen, C. N., Chen, H. S., \& Hsu, H. C. (2020). Maternal prepregnancy body mass index, gestational weight gain, and risk of adverse perinatal outcomes in Taiwan: A population-based birth cohort study. International Journal of Environmental Research and Public Health, 17(4), 1-11.

Chen, Z., Du, J., Shao, L., Zheng, L., Wu, M., Ai, M., \& Zhang, Y. (2010). Prepregnancy body mass index, gestational weight gain, and pregnancy outcomes in China. International Journal of Gynecology and Obstetrics, 109(1), 41-44.

Cnattingius, S., \& Lambe, M. (2002). Trends in Smoking and Overweight During Pregnancy: Prevalence, Risks of Pregnancy Complications, and Adverse Pregnancy Outcomes. Seminars in Perinatology, 26(4), 286-295.

Dell"osbel, R. S., Cremonese, C., \& Gregoletto, M. L. (2019). Ganho de Peso Gestacional e Fatores Associados em Gestantes e Recém-Nascido. Revista Contexto \& Saúde, 19(37), 20-29.

Deputy, N. P., Dub, B., \& Sharma, A. J. (2018). Morbidity and Mortality Weekly Report Prevalence and Trends in Prepregnancy Normal Weight-48 States, New York City, and District of Columbia. Morbidity and Mortality Weekly Report, 66(51), 1402-1404.

Djelantik, A., Kunst, A., Wal, M. v., Smit, H., \& Vrijkotte, T. (2011). Contribution of overweight and obesity to the occurrence of adverse pregnancy outcomes in a multi-ethnic cohort: Population attributive fractions for Amsterdam. BJOG An International Journal of Obstetrics and Gynaecology, 119(3), 283-290.

Dodd, J. M., Grivell, R. M., Nguyen, A.-M., Chan, A., \& Rovinson, J. S. (2011). Maternal and perinatal health outcomes by body mass index category. Australian and New Zealand Journal of Obstetrics and Gynaecology, 51(2), 136-140.

Ferraz, L., Albiero C., Boechat S.G., Fonseca, I.P., Farias, V.P., Braga, A., \& Lopes, P.F. (2018). Micronutrientes e sua importância no período gestacional. Revista Saber Científico, 7(1), 68-82.

Ferreira, L. A., Piccinato, C. d., Cordioli, E., \& Zlotnik, E. (2019). Pregestational body mass index, weight gain during pregnancy and perinatal outcome: a retrospective descriptive study. Einstein (São Paulo), 18, 1-6.

Gonçalves, A.S.A., Costa, E.J., Souza, E.O., Campos, E.S., Silva, F., Paiva, A.A. (2019). Perfil alimentar nutricional durante a gestação. Mosrtra de trabalhos do curso de nutrição da UNIVAG, 4, 106-120.

Gonçalves, C. V., Mendoza-Sassi, R. A., Cesar, J. A., Castro, N. B., \& Bortolomedi, A. P. (2012). Body mass index and gestational weight gain as factors predicting complications and pregnancy outcome. Rev Bras Ginecol Obstet, 34(7), 305-309.

Han, Y., Ha, E., Park, H., Kim, Y., \& Lee, S. (2011). Relationships betweem pregnancy outcomes, biochemical markres and pre-pregnancy body mass index. International Journal of Obesity, 35(4), 570-577.

Hancke, K., Gundelach, T., Hay, B., Sander, S., Reister, F., \& Weiss, J. M. (2015). Pre-pregnancy obesity compromises obstetric and neonatal outcomes. Journal of Perinatal Medicine, 43(2), 141-146.

IBGE. (2019). Instituto Brasileiro de Geografia e Estatística. Fonte: https://biblioteca.ibge.gov.br/index.php/biblioteca-catalogo?view=detalhes\&id=2101758

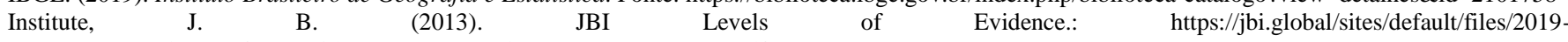
05/JBI\%20Levels\%20of\%20Evidence\%20Supporting\%20Documents-v2.pdf

Jenabia, E., \& AslToghiria, M. (2011). The effect of body mass index on delivery outcomes. Procedia - Social and Behavioral Sciences, 28,465 - 469.

Kim, S., Mendola, P., Zhu, Y., Hwang, B., \& Grantz, K. (2017). Spontaneous and indicated preterm delivery risk is increased among overweight and obese women without prepregnancy chronic disease. BJOG: International Journal of Obstetrics and Gyneacology, 124(11), 1708-1716.

Konje, J., Imrie, A., \& Hay, D. M. (2016). Pregnancy in obese women. Journal of Obstetrics and Gynaecology, 13(6), 413-418.

Lana, T. C., Oliveira, L. V., Martins, E. F., Santos, N. C., Matozinhos, F. P., \& Mendes, M. S. (2020). Prevalência, fatores associados e desfechos reprodutivos relacionados ao ganho de peso gestacional excessivo. Rev enferm UERJ, 28, 1-8.

Lima, A. P., Castral, T. C., Leal, L. P., Javorski, M., Sette, G. C., Scochi, C. G., \& Vasconcelos, M. G. (2019). Aleitamento materno exclusivo de prematuros e motivos para sua interrupção no primeiro mês pós-alta hospitalar. Revista Gaucha de Enfermagem, 40, 1-8.

Lutsiv, O., Mah, J., Beyene, J., \& McDonald, S. D. (2015). The effects of morbid obesity on maternal and neonatal health outcomes: a systematic review and meta-analyses. obesity reviews, 16(7), 531-546.

Lynch, A. M., Hart, J. E., Agwu, O. C., Fisher, B. M., West, N. A., \& Gibbs, R. S. (2014). Association of extremes of prepregnancy BMI with the clinical presentations of preterm birth. American journal of obstetrics and gynecology, 210(5).

Lynch, T. A., Malshe, A., Colihan, S., Meyers, J., Li, D., Holloman, C., . . Olson-Chen, C. (2019). Impact of Maternal Obesity on Perinatal Outcomes in Preterm Prelabor Rupture of Membranes $\geq 34$ Weeks. Am J Perinatol, 37(5), 467-474.

Madan, J., Chen, M., Goodman, E., Davis, J., Allan, W., \& Dammann, O. (2010). Maternal obesity, gestational hypertension, and preterm delivery. The Journal of Maternal-Fetal and Neonatal Medicine, 23(1), 82-88.

Manera, F., \& Höfelmann, D. A. (2017). Excesso de peso em gestantes acompanhadas em unidades de saúde de Colombo, Paraná, Brasil. DEMETRA, 14, 116.

Marchi, J., Berg, M., Dencker, A., Olander, E. K., \& Begley, C. (2015). Risks associated with obesity in pregnancy, for the mother and baby: A systematic review of reviews. obesity reviews, 16(8), 621-638.

Masho, S. W., Bishop, D. L., \& Munn, M. (2013). Pre-pregnancy BMI and weight gain: where is the tipping point for preterm birth? BMC Pregnancy and Childbirth, 13(1), 1-10. 
Melchor, I., Burgos, J., Campo, A. d., Aiartzaguena, A., Gutiérrez, J., \& Melchor, J. C. (2019). Effect of maternal obesity on pregnancy outcomes in women delivering singleton babies: a historical cohort study. J. Perinat. Med, 1-6.

Nascimento, I. B., Dienstman, G., Souza, M. L., Silva, T. R., Fleig, R., \& Silva, J. C. (3 de Março de 2018). . Dyslipidemia and maternal obesity: Prematurity and neonatal prognosis. Scielo, 63.

Oliveira, A. C., Pereira, L. A., Ferreira, R. C., \& Clemente, A. P. (2018). Estado nutricional materno e sua associação com o peso ao nascer em gestações de alto risco. Ciência \& Saúde Coletiva, 23(7), 2373-2382.

Otaviano, M. D., Monteiro, M. A., Santos, F. J., Moraes, A. L., Alves, T. d., Sousa, M. G., \& Erandielly, F. (2021). Perfil de mães de recém-nascidos prétermo em um município do semiárido cearense. Research, Society and Development, 10(3), 1-10.

Palatnik, A., Miller, E. S., Son, M., \& Kominiarek, M. A. (2016). Association among Maternal Obesity, Cervical Length, and Preterm Birth. American Journal of Perinatology, 34(5), 471-479.

Papachatzi, E., Paparrodopoulos, S., Papadopoulos, V., Dimitriou, G., \& Vantarakis, A. (2016). Pre-pregnancy maternal obesity in Greece: A case-control analysis. Early Human Development, 93, 57-61.

Parveen, M., Ismaile, M., \& Massod, R. (2017). Obesity as a Predictor of Adverse Maternal Outcomes among Pregnant Women of a rural community. $P$ J M HS, 11(3), 1-4.

Pedrine, D. B., Cunha, M. L., \& Breigeiron, M. K. (2020). Maternal nutritional status in diabetes mellitus and neonatal characteristics at birth. Rev Bras. Enferm, 73, 1-6.

Pires, C.C., Rodrigues, M.L., Capeli, J.C.S., Santos, M.M.A.S., \& Baião, M.R. (2018). O excesso de peso na perspectiva de gestantes assistidas na atenção primária à saúde de um município da região do Norte Fluminense. Demetra, 13(3), 555-568.

Poston, L., \& Rishi Caleyachetty, S. C. (2016). Preconceptional and maternal obesity: epidemiology and health consequences. Lancet DIabetes-Endocrinol, 4(12), 1025-1036.

Puscasi, L., Roman, H., Newman, R., Hulsey, T. F., Hulsey, T. C., \& Mircea, O. (2013). Association of pre-pregnant body mass index and gestational weight gain with the timing of deliveryand fetal growth in singletons. Obstetrics, 9(34), 161-166.

Rafei, R. E., Abbas, H. A., Charafeddine, L., Nakad, P., Bizri, A. A., Hamod, D., \& Yunis, K. A. (2016). Association of Pre-Pregnancy Body Mass Index and Gestational Weight Gain with Preterm Births and Fetal Size: An Observational Study from Lebanon. Paediatric and Perinatal Epidemiology, $30(1)$, 38-45.

Rodrigues, J. C. (2018). Desenvolvimento inicial de bebês nascidos pré-termo de alto risco neonatal em comparação a bebês nascidos a termo. Dissertação de Mestrado. Faculdade de Filosofia, Ciências e Letras de Ribeirão Preto. Área do Conhecimento Psicologia em Saúde e Desenvolvimento.

Savitz, D. A., Stei, C. R., A. M.-R., \& Herring, A. H. (2011). Gestational Weight Gain and Birth Outcome in Relation to Prepregnancy Body Mass Index and Ethnicity. Ann Epidemiol, 21(2), 78-85.

Schieve, L. A., Cogswell, M. E., Scanlon, K. S., Perry, G., Ferre, C., Blackmore-Prince, C., . . Rosenberg, D. (2000). Prepregnancy Body Mass Index and Pregnancy. Obstetrics \& Gynecology, 96(2), 194-200.

Silva, R. M. (2019). O cuidado de crianças prematuras em região de fronteira: necessidades essenciais e especiais de saúde. Tese de Doutorado, Escola de Enfermagem de Ribeirão Preto, Universidade de São Paulo, Ribeirão Preto.

Soares, N. P., Resende, R. A., Augusto, M., Aires, M., \& Corrêa, I. B. (2020). Incopetência istmo cervical: Indicação e técnica da cerclagem de emergência. Revista de Patologia do Tocantins, 7(2), 34-37.

Sousa, T. M., Melleiro, V., Silva, G., SIlva, L. R., Calandrino, L., Fukuda, Y., . . Augusto, M. (2020). Desfechos neonatais na pré eclâmpsia: Correlação com fatores clínicos e laborais materno. Revista UNILUS Ensino e Pesquisa, 17(47), 55-69.

Souza, A. P., Landmann, C., \& Nogueira, G. (2019). Prevalence of obesity and associated factors in the Brazilian population: a study of data from the 2013 National Health Survey. REV BRAS EPIDEMIOL, 22(1), 1-14.

Souza, S. d., Silva, A. B., Cavalcante, U. M., Lima, C. M., \& Souza, T. C. (2018). Adult obesity in different countries: an analysis via beta regression models. CSP Reports in Public Health, 1-13.

Su, X. J., Huang, S. J., Li, X., \& Du, Q. L. (2020). Prepregnancy Overweight and Obesity Are Associated with an Increased Risk of Preterm Birth in Chinese Women. Obesity Facts, 13(2), 237-244.

Torloni, M. R., Betran, A. P., Silvia Daher, M. W., Dolan, S. M., Menon, R., Bergel, E., \& Tomas Allen, M. B. (2010). Maternal BMI and preterm birth: A systematic review of the literature with meta-analysis. The Journal of Maternal-Fetal \& Neonatal Medicine, 22(11), 957-970.

Tricco, A. C., Lillie, E., Zarin, W., O'Brien K. K., C. H., D, L., D, M., . . . Lewin, S. (2018). PRISMA Extension for Scoping Reviews (PRISMA-ScR): Checklist and Explanation. Annals of Internal Medicine, 167(7), 467-463.

Wise, L. A., Palmer, J. R., Heffner, L. J., \& Rosenberg, L. (2010). Prepregnancy Body Size, Gestational Weight Gain, and Risk. Epidemiology, 21(2), 243252 .

Z, Y., H, P., L, F., R, S., A, R., \& P, K. (2020). Contribution of maternal overweight and obesity to the occurrence of adverse pregnancy outcomes. Obstetric Anesthesia Digest, 40(1), 34-35. 
Research, Society and Development, v. 10, n. 13, e180101320971, 2021

(CC BY 4.0) | ISSN 2525-3409 | DOI: http://dx.doi.org/10.33448/rsd-v10i13.20971

Zhong, Y., Cahill, A. G., Macones, G. A., Zhu, F., \& Odibo, A. O. (2010). The Association between Prepregnancy Maternal Body Mass Index and Preterm Delivery. American Journal of perinatology, 27(4), 293-298.

Zhou, Y., Li, H., Zhang, Y., Zhang, L., Liu, J., \& Liu, J. (2019). Association of Maternal Obesity in Early Pregnancy with Adverse Pregnancy Outcomes: A Chinese Prospective Cohort Analysis. Obesity, 27, 1030-1036. 